\title{
Load weight estimation on an excavator in static and dynamic motions
}

\author{
Mehmet Ferlibas and Reza Ghabcheloo \\ Faculty of Engineering and Natural Sciences (ENS), Tampere University, Tampere, Finland \\ E-mail: mehmet.ferlibas@gmail.com,reza.ghabcheloo@tuni.fi
}

\begin{abstract}
Excavators are one of the commonly used types of hydraulic machines in earth moving operations. The material handled is often transferred by dump trucks having a payload capacity that should not be exceeded. Payload monitoring systems are needed in order to prevent the possible problems during the delivery, increase the work efficiency, reduce the cost, and obtain the product information automatically without the requirement of truck scales. In this study, we propose a novel approach to estimate the load weight in the bucket of the excavator when the machine links are in motion. We consider the excavator as a three-revolute joint manipulator in vertical plane with the boom, the stick, and the bucket links. We rewrite the dynamic torque equations in a decoupled form as the linear combination of dynamic parameters and functions of joint angles, velocities, and accelerations. We perform least squares estimation to identify these parameters allowing us to predict the no load joint torques for any configuration of the links. We show that the most accurate torque prediction is the difference between the boom torque and the stick torque. We then derive the relation between the joint torques with and without the load, which are functions of the dynamic parameters. Using these equations, we can estimate the load weight. The relation becomes simpler when the links are stationary, since only the gravitational parameters remain present in the torque equations. The relation in dynamic case requires the parameters of the polar coordinates for the center of gravity of the bucket and we show that these parameters can be estimated with the knowledge of the empty bucket mass. We summarize our findings on load weight estimation for different cases including stationary poses and dynamic trajectories on free space and discuss the results. Although the friction is neglected throughout the modeling, the results obtained indicate that the effect of the static friction plays an important role in the accuracy of the estimated payload mass. We show that our dynamic model based solution is very promising, and exhibit only $2 \%$ error for high enough velocities.
\end{abstract}

Keywords: Load weight estimation, payload estimation, dynamic parameter estimation, hydraulic machine, excavator

\section{Introduction}

Excavators play a significant role in variety of work sectors such as construction industry, mining, forestry activities and agriculture. The weight of the payload on the dump trucks has to be measured since each truck has a payload capacity that should not be exceeded for safety reasons. However, the use of truck scales increase the cost, and they are not always available in every kind of work sites. Therefore, there is a need for payload monitoring systems for excavators, in order to increase the work efficiency, lower the operational cost, and eliminate the need for truck scales and automatically calculate the amount of work done. [1].

Different research works have been done in order to develop such payload monitoring systems for different hydraulically operating machines. There are existing methods proposed for wheel-loaders that use a neural network approach [2] or generate the dynamic model of the wheel loader [3]. Also, there are algorithms developed for mini excavators [4] and excavators [1,5-8].

A research on available payload estimation systems for excavators have been presented in [9] and stated that the load weight is calculated by making a linear interpolation using a map consisting of the data with two different 
load weights on a pre-defined range of motion. A method based on the static parameters of the dynamic model of the excavator is developed in [4] for stationary poses. An analytical approach for payload estimation has been presented in [8] for simulation environment and it is validated on a real excavator in [7].

In order to identify the dynamic parameters of the dynamic model of the excavators, the structural similarity of the excavators to the serial link robot manipulators has been benefited. Since the excavator links, the boom, the stick, and the bucket operate with the help of hydraulic actuators, change in the joint angles have been described as functions of the linear displacement in these hydraulic actuators [10].

Problem of parameter estimation on excavators has been studied in different research works. Inertial parameters are identified by making use of the dynamic model of the excavator with the displacement measurements of the hydraulic cylinders and pressure readings in [11]. The link parameter values and friction terms have been estimated in [12]. The dynamic parameters, gravitational parameters and Coulomb \& Viscous friction parameters were estimated in $[4,13,14]$ as these parameters are necessary to develop weighing algorithms.

In this paper, a load weight estimation method is developed. The problem is divided into two parts as static estimation and dynamic estimation. The static approach that is developed using a mini-excavator in [4] is tested using a larger scale Komatsu 138PC-US8 excavator and the results show that the effect of static friction has a significant effect on the estimated load masses. The dynamic weighing algorithm is developed assuming that the bucket's center of gravity is independent from the variable load weight in the bucket. The method proposed requires the dynamic parameter estimation process so that the no-load torque values could be predicted accurately as these predictions were needed in order to estimate the load mass.

The structure of this paper is as follows. Machine instrumentation is explained in the section 2 and the dynamic model of the excavator is derived in the section 3. The static estimation and dynamic estimation and the results obtained are discussed in the sections 4 and 5, respectively. Finally, the conclusion is given in the section 6.

\section{Machine instrumentation}

A Komatsu PC138US-8 excavator was used in data collection for this research. The CAD model of the excavator can be seen in the figure 1 below. The excavator is taken into account as a three-revolute joint manipulator in vertical plane with the boom, the stick and the bucket links. There are four IMU sensors installed on the cabin frame, the boom link, the stick link, and the bucket that is the end-effector in order to measure the angular position and angular velocity values of each link. The angular position measurements of each link are recorded with respect to the previous link. In other words, angular position of the bucket is recorded with respect to the stick, angular position of the stick is recorded with respect to the boom, and angular position of the boom is recored with respect to the cabin frame. Furthermore, there are two pressure sensors located in each hydraulic cylinder. Therefore, there are six pressure sensors used in total, to measure the pressures inside the chambers and to calculate the actuator forces. All the sensors used operate at frequency of $200 \mathrm{~Hz}$.

The boom, stick and bucket angular positions are shown in the figure 1 as $\theta_{2}, \theta_{3}$, and $\theta_{4}$, respectively. The constant angles $\beta_{1}$ and $\beta_{2}$ are used to calculate the joint variable $q_{2}$ and the constant angles $\beta_{3}$ and $\beta_{4}$ are used to obtain the joint variable $q_{3}$. The constant lengths $L_{11}$ and $L_{12}$ from boom joint to the both ends of the hydraulic actuator are used together with the joint variable $q_{2}$ in order to obtain the actuator length $z_{2}$. Similarly, the constant lengths $L_{21}$ and $L_{22}$ are used together with the joint variable $q_{3}$ to calculate the actuator length $z_{3}$. The actuator lengths are used in calculation of cylinder Jacobians that is needed for calculating the torques exerted in each link. Since the IMU sensor on the bucket was located on the dog bone, obtaining the joint variable $q_{4}$ from the joint angle $\theta_{4}$ is more difficult. The dimensions of the four-bar linkage were needed in order to convert the joint angle $\theta_{4}$ to the joint variable $q_{4}$. Note that the index 1 is reserved for the cabin frame which is kept outside the scope of this study.

Torque calculation from the pressure readings and cylinder jacobians that are obtained using the angular position measurements has been discussed in [1].

The simplest schematic diagram of the excavator is illustrated in the figure 2 . The boom torque, the stick torque, and the bucket torque are represented as $\tau_{2}, \tau_{3}$, and $\tau_{4}$, respectively. The parameters $\alpha_{i}$ and $r_{i}$ are the polar coordinates of the center of gravity $\left(\mathrm{cg}_{i}\right)$ of link $\mathrm{i}$. The angular position of the links are $\theta_{2}$ for the boom, $\theta_{23}=$ $\theta_{2}+\theta_{3}$ for the stick, and $\theta_{234}=\theta_{2}+\theta_{3}+\theta_{4}+c$ for the bucket with respect to the horizontal plane, where $c$ is a constant angle, that is shown in the figure 1, used in order to map the bucket angular position measurement to the bucket tip. Derivation of the constant angle $c$ is discussed in [1].

\section{Dynamic model of the excavator}

Excavators are hydraulically actuating manipulators. Excluding the cabin frame from our research, and assuming that the tiltrotator is only a series of offsets and nonfunctional, the excavator can be considered as a planar three- 


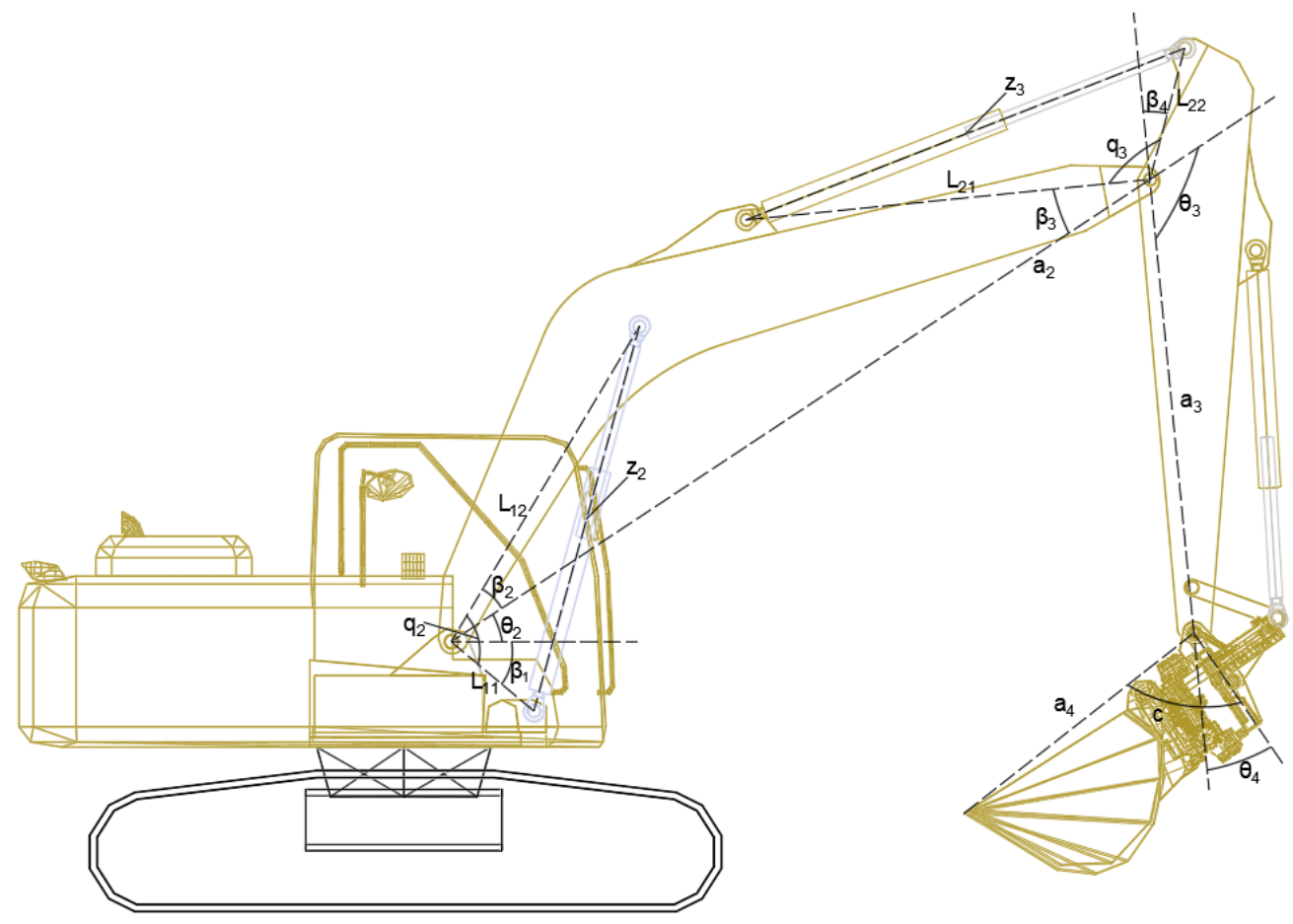

Figure 1: Excavator CAD Model [1]

revolute joint manipulator [13].

The following equation (1) describes the dynamic model of a robot manipulator [6,15-17]:

$$
\tau=D(\Theta) \ddot{\Theta}+C(\Theta, \dot{\Theta}) \dot{\Theta}+G(\Theta)
$$

Where,

- $\tau$ is the joint torque vector,

- $\Theta$ is the vector of joint angles,

- $D(\Theta)$ is the inertia matrix,

- $C(\Theta, \dot{\Theta}) \dot{\Theta}$ is the vector of Coriolis and centrifugal terms,

- $\mathrm{G}(\Theta)$ is the gravity torque vector.

As pointed out in [17], the torque equation (1) can be written as the linear combination of dynamic parameters that are assumed to be constant, $\pi$, and a matrix of functions of joint positions, velocities and accelerations $Y(\Theta, \dot{\Theta}, \ddot{\Theta})$ :

$$
\tau=Y(\Theta, \dot{\Theta}, \ddot{\Theta}) \pi
$$

Neglecting the friction, we can assume that the system is conservative, i.e. the summation of kinetic energy and potential energy is constant, and keeping the cabin frame stationary, the torque equations (3) were obtained using Euler-Lagrange method $[13,14]$ : 


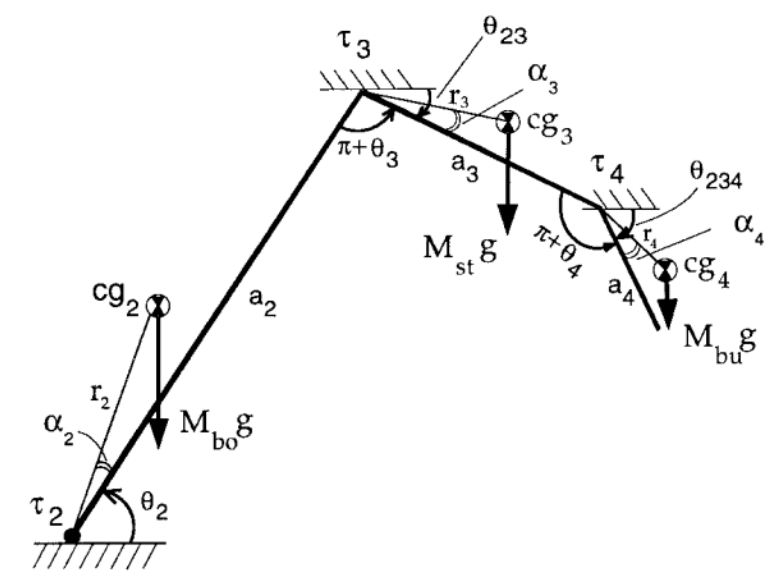

Figure 2: Excavator schematic diagram [13]

$$
\begin{aligned}
\tau_{4}= & \left(I_{b u}+M_{b u} r_{4}^{2}\right) \ddot{\theta}_{234}+M_{b u} a_{2} r_{4}\left[\ddot{\theta}_{2} \cos \left(\theta_{34}+\alpha_{4}\right)+\dot{\theta}_{2}^{2} \sin \left(\theta_{34}+\alpha_{4}\right)\right] \\
& +M_{b u} a_{3} r_{4}\left[\ddot{\theta}_{23} \cos \left(\theta_{4}+\alpha_{4}\right)+\dot{\theta}_{23}^{2} \sin \left(\theta_{4}+\alpha_{4}\right)\right]+M_{b u} g r_{4} \cos \left(\theta_{234}+\alpha_{4}\right) \\
\tau_{3}= & \tau_{4}+\left(I_{s t}+M_{s t} r_{3}^{2}+M_{b u} a_{2}^{3}\right) \ddot{\theta}_{23}+M_{b u} a_{2} a_{3}\left(\ddot{\theta}_{2} \cos \left(\theta_{3}\right)+\dot{\theta}_{2}^{2} \sin \left(\theta_{3}\right)\right) \\
& +M_{b u} a_{3} r_{4}\left[\ddot{\theta}_{234}\left(\theta_{4}+\alpha_{4}\right)-\dot{\theta}_{234}^{2} \sin \left(\theta_{4}+\alpha_{4}\right)\right] \\
& +M_{s t} a_{2} r_{3}\left[\ddot{\theta}_{2} \cos \left(\theta_{3}+\alpha_{3}\right)+\dot{\theta}_{2}^{2} \sin \left(\theta_{3}+\alpha_{3}\right)\right] \\
& +M_{b u} g a_{3} \cos \left(\theta_{23}\right)+M_{s t} g r_{3} \cos \left(\theta_{23}+\alpha_{3}\right) \\
\tau_{2}= & \tau_{3}+\left[I_{b o}+M_{b o} r_{2}^{2}+\left(M_{s t}+M_{b u}\right) a_{2}^{2}\right] \ddot{\theta}_{2} \\
& +M_{s t} a_{2} r_{3}\left[\ddot{\theta}_{23} \cos \left(\theta_{3}+\alpha_{3}\right)-\dot{\theta}_{23}^{2} \sin \left(\theta_{3}+\alpha_{3}\right)\right] \\
& +M_{b u} a_{2} a_{3}\left(\ddot{\theta}_{23} \cos \left(\theta_{3}\right)-\dot{\theta}_{23}^{2} \sin \left(\theta_{3}\right)\right. \\
& +M_{b u} a_{2} r_{4}\left[\ddot{\theta}_{234} \cos \left(\theta_{34}+\alpha_{4}\right)-\dot{\theta}_{234}^{2} \sin \left(\theta_{34}+\alpha_{4}\right)\right] \\
& +\left(M_{b u}+M_{s t}\right) g a_{2} \cos \left(\theta_{2}\right)+M_{b o} g r_{2} \cos \left(\theta_{2}+\alpha_{2}\right)
\end{aligned}
$$

Where $I_{b o}, I_{s t}$, and $I_{b u}$ are the moments of inertia of the boom link, the stick link, and the bucket link, respectively and the parameters $M_{b o}, M_{s t}$, and $M_{b u}$ represent the masses of the boom link, the stick link and the bucket link, respectively. The parameter $a_{2}$ is the linear distance between the boom joint and the stick joint. Similarly, the parameter $a_{3}$ is the linear displacement between the stick joint and the bucket link, and the parameter $a_{4}$ is the distance from the bucket link to the bucket tip.

\section{Static estimation of the load weight}

The torque equations given in the equation (3) are reduced to the following equation (4) when the machine links are stationary, i.e. all the velocity and acceleration terms are set to zero:

$$
\begin{aligned}
& \tau_{4}=M_{b u} g r_{4} \cos \left(\theta_{234}+\alpha_{4}\right) \\
& \tau_{3}=\tau_{4}+M_{b u} g a_{3} \cos \left(\theta_{23}\right)+M_{s t} g r_{3} \cos \left(\theta_{23}+\alpha_{3}\right) \\
& \tau_{2}=\tau_{3}+\left(M_{b u}+M_{s t}\right) g a_{2} \cos \left(\theta_{2}\right)+M_{b o} g r_{2} \cos \left(\theta_{2}+\alpha_{2}\right)
\end{aligned}
$$

We rewrite the static torque equation (4) above in the decoupled form, that is the torque difference of the two consecutive joints and benefit from the well-known trigonometric identity, $\cos (\alpha+\beta)=\cos (\alpha) \cos (\beta)-\sin (\alpha) \sin (\beta)$, and obtain the following equation (5):

$$
\begin{aligned}
\tau_{4} & =M_{b u} g r_{4}\left[\cos \left(\theta_{234}\right) \cos \left(\alpha_{4}\right)-\sin \left(\theta_{234}\right) \sin \left(\alpha_{4}\right)\right] \\
\tau_{34}=\tau_{3}-\tau_{4} & =M_{b u} g a_{3} \cos \left(\theta_{23}\right)+M_{s t} g r_{3}\left[\cos \left(\theta_{23}\right) \cos \left(\alpha_{3}\right)-\sin \left(\theta_{23}\right) \sin \left(\alpha_{3}\right)\right] \\
\tau_{23}=\tau_{2}-\tau_{3} & =\left(M_{b u}+M_{s t}\right) g a_{2} \cos \left(\theta_{2}\right)+M_{b o} g r_{2}\left[\cos \left(\theta_{2}\right) \cos \left(\alpha_{2}\right)-\sin \left(\theta_{2}\right) \sin \left(\alpha_{2}\right)\right]
\end{aligned}
$$


The equation (5) can be converted into matrix form as stated in the equation (2):

$$
\left[\begin{array}{c}
\tau_{4} \\
\tau_{34} \\
\tau_{23}
\end{array}\right]=g\left[\begin{array}{cccccc}
\cos \left(\theta_{234}\right) & -\sin \left(\theta_{234}\right) & 0 & 0 & 0 & 0 \\
0 & 0 & \cos \left(\theta_{23}\right) & -\sin \left(\theta_{23}\right) & 0 & 0 \\
0 & 0 & 0 & 0 & \cos \left(\theta_{2}\right) & -\sin \left(\theta_{2}\right)
\end{array}\right]\left[\begin{array}{c}
\pi_{s 1} \\
\pi_{s 2} \\
\pi_{s 3} \\
\pi_{s 4} \\
\pi_{s 5} \\
\pi_{s 6}
\end{array}\right]
$$

Where the vector $\left[\begin{array}{llllll}\pi_{s 1} & \pi_{s 2} & \pi_{s 3} & \pi_{s 4} & \pi_{s 5} & \pi_{s 6}\end{array}\right]^{T}$ is named as the vector of gravitational parameters, $\pi_{s}$ :

$$
\pi_{s}=\left[\begin{array}{c}
\pi_{s 1} \\
\pi_{s 2} \\
\pi_{s 3} \\
\pi_{s 4} \\
\pi_{s 5} \\
\pi_{s 6}
\end{array}\right]=\left[\begin{array}{c}
M_{b u} r_{4} \cos \left(\alpha_{4}\right) \\
M_{b u} r_{4} \sin \left(\alpha_{4}\right) \\
M_{b u} a_{3}+M_{s t} r_{3} \cos \left(\alpha_{3}\right) \\
M_{s t} r_{3} \sin \left(\alpha_{3}\right) \\
\left(M_{b u}+M_{s t}\right) a_{2}+M_{b o} r_{2} \cos \left(\alpha_{2}\right) \\
M_{b o} r_{2} \sin \left(\alpha_{2}\right)
\end{array}\right]
$$

Note that the equation (6) is linear in the gravitational parameters. Once the torque vector $\left[\tau_{4}, \tau_{34}, \tau_{23}\right]^{T}$ is obtained, the vector of gravitational parameters, $\pi_{s}$, can be found using Least Squares Estimation (LSE) as explained in the section 4.1.

The pressure readings have been used to calculate the force $(\mathrm{F})$ exerted on each hydraulic actuator using the relation $F=P_{1} A_{1}-P_{2} A_{2}$, where $P_{1}$ and $P_{2}$ are the pressures in chamber $\mathrm{A}$ and chamber $\mathrm{B}$ of the hydraulic cylinders and $A_{1}$ and $A_{2}$ are the cross-sectional areas of chamber $\mathrm{A}$ and chamber $\mathrm{B}$, respectively.

The torque values for each joint has been calculated with the multiplication of the forces and cylinder jacobians. Figure 3 visualizes the constant $\beta_{1}$ and $\beta_{2}$ angles that are used to calculate the boom joint variable, $q_{2}$, together with the measured boom angle, $\theta_{2}$, the constant lengths from boom joint to the both ends of the hydraulic cylinder are represented as $L_{11}$ and $L_{12}$ that are used to calculate the length of the actuator, $z_{2}$. The equation (8) shows the calculation of the boom cylinder jacobian, $J_{b o}$. Similarly, how the stick cylinder jacobian and the bucket cylinder jacobian are derived can be seen in [1].

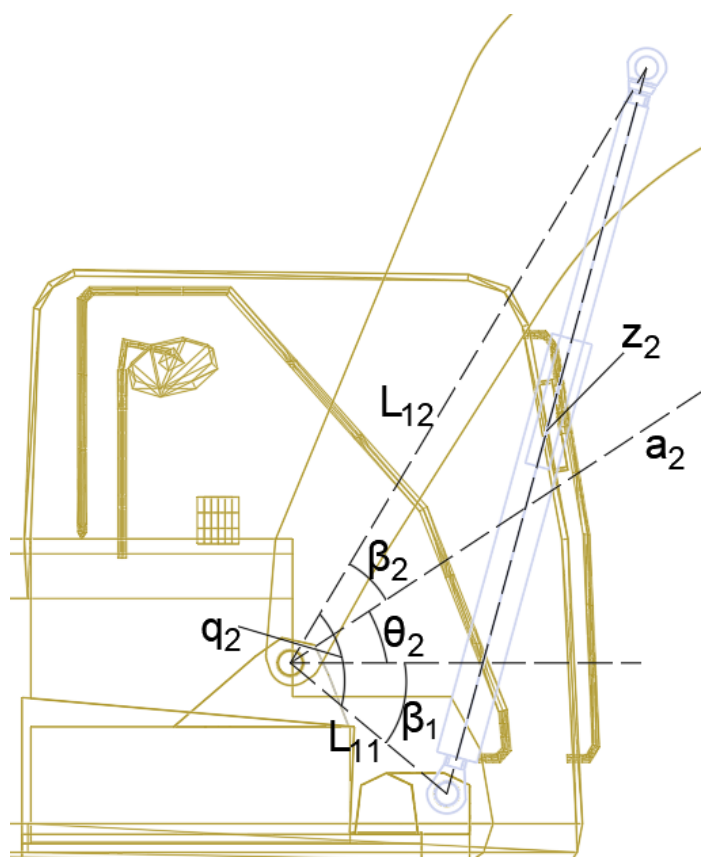

Figure 3: Boom joint angle and joint variable [1] 


$$
\begin{aligned}
q_{2} & =\beta_{1}+\beta_{2}+\theta_{2} \\
z_{2} & =\sqrt{L_{11}^{2}+L_{12}^{2}-2 L_{11} L_{12} \cos \left(q_{2}\right)} \\
J_{b o} & =\frac{d z_{2}}{d \theta_{2}}=\frac{L_{11} L_{12} \sin \left(q_{2}\right)}{z_{2}}
\end{aligned}
$$

After obtaining the torque values, the gravitational parameters can be found using the following equation (9):

$$
\pi=\left(Y(\Theta)^{T} Y(\Theta)\right)^{-1} Y(\Theta)^{T} \tau
$$

Where $Y(\Theta)$ is the regressor matrix, and defined as follows:

$$
Y(\Theta)=g\left[\begin{array}{cccccc}
\cos \left(\theta_{234}\right) & -\sin \left(\theta_{234}\right) & 0 & 0 & 0 & 0 \\
0 & 0 & \cos \left(\theta_{23}\right) & -\sin \left(\theta_{23}\right) & 0 & 0 \\
0 & 0 & 0 & 0 & \cos \left(\theta_{2}\right) & -\sin \left(\theta_{2}\right)
\end{array}\right]
$$

\subsection{Static parameter estimation}

The estimation of the gravitational parameters that are given in the equation (7) allows us to predict the torque values in decoupled form, when the bucket of the excavator is empty.

In order to obtain the gravitational parameters, a training data set consisting of 38 different static postures of the machine links has been collected. In the data set, angular positions of the boom link, the stick link and the bucket link has been recorded together with the pressure readings of the hydraulic cylinders.

After gathering the data and making use of the equation (10), the gravitational parameters are estimated as given in the table 1 .

\section{Table 1: Estimated gravitational parameters}

\begin{tabular}{cc}
\hline Parameter & Estimated Value (kgm) \\
\hline$\pi_{s 1}$ & 189.85 \\
$\pi_{s 2}$ & 318.80 \\
$\pi_{s 3}$ & 2753.27 \\
$\pi_{s 4}$ & 132.33 \\
$\pi_{s 5}$ & 8297.89 \\
$\pi_{s 6}$ & 1156.44 \\
\hline
\end{tabular}

As can be seen from the table 1, all of the parameters are found to be positive, as expected.

The estimated gravitational parameters have been tested on a test data set consisting of 19 different static postures that are different than the ones in the training data set. The mean absolute percentage value of the error (MAPE) in the predicted torque values are reported in the table 2 below:

Table 2: Accuracy of predicted no-load torque values

\begin{tabular}{cc}
\hline Predicted Torque & MAPE \\
\hline$\tau_{4}$ & $40.98 \%$ \\
$\tau_{34}$ & $10.25 \%$ \\
$\tau_{23}$ & $8.31 \%$ \\
\hline
\end{tabular}

From the results presented in the table 2 , we conclude that the most accurate torque prediction is $\tau_{23}$, that is the difference between the boom torque and the stick torque. The highest error has been obtained for the bucket toruqe, $\tau_{4}$, and the possible reasons of the error are listed below:

- The effect of static friction is more dominant compared to other torque values,

- The test data of the bucket angular measurements exceeds the range of the ones in the training data. 


\subsection{Load weight estimation using the gravitational parameters}

For an arbitrary mass in the bucket of the excavator, $\hat{M}$, the static torque equations given in the equation (5) can be rewritten by replacing $M_{b u}$ with $M_{b u}+\hat{M}$ and the following equations (11) can be obtained:

$$
\begin{aligned}
\tau_{4} & =\left(M_{b u}+\hat{M}\right) g r_{4} \cos \left(\theta_{234}+\alpha_{4}\right) \\
\tau_{34} & =\left(M_{b u}+\hat{M}\right) g a_{3} \cos \left(\theta_{23}\right)+M_{s t} g r_{3} \cos \left(\theta_{23}+\alpha_{3}\right) \\
\tau_{23} & =\left(M_{b u}+\hat{M}+M_{s t}\right) g a_{2} \cos \left(\theta_{2}\right)+M_{b o} r_{2} \cos \left(\theta_{2}+\alpha_{2}\right)
\end{aligned}
$$

These torque equations given in the equation 11 are valid for any value of the load in the bucket and can be written as the summation of the no load torque and the torque due to the load in the bucket as stated in the following equation (12) [4]:

$$
\begin{aligned}
\tau_{4_{L}} & =\tau_{4_{N L}}+\hat{M} g r_{4} \cos \left(\theta_{234}+\alpha_{4}\right) \\
\tau_{34_{L}} & =\tau_{34_{N L}}+\hat{M} g a_{3} \cos \left(\theta_{23}\right) \\
\tau_{23_{L}} & =\tau_{23_{N L}}+\hat{M} g a_{2} \cos \left(\theta_{2}\right)
\end{aligned}
$$

In the equation (12) above, the subscript NL represents the no load torque value and the subscript L denotes the torque with the load. We can predict the no load torque values by making use of the estimated gravitational parameters. In the light of the results presented in the table 2, the most suitable torque equation is the difference between the boom torque and the stick torque, $\tau_{23}$. We can predict the value of $\tau_{23}$ by making use of the last two elements of the gravitational parameter vector, $\pi_{s 5}$, and $\pi_{s 6}$ and solve the equation 12 for $\hat{M}$ as in the equation (13) below [4]:

$$
\hat{M}=\frac{\tau_{23_{L}}-\tau_{23_{N L}}}{g a_{2} \cos \left(\theta_{2}\right)}
$$

In order to validate the usability of this approach presented above, we collected data sets with two different reference load masses that are $250 \mathrm{~kg}$ and $500 \mathrm{~kg}$. The collected data sets have 37 different static postures for $250 \mathrm{~kg}$ and 42 different static postures for $500 \mathrm{~kg}$. The results of the estimated load weight are presented in the table 3 below:

Table 3: The results of static load weight estimation using the estimated gravitational parameters

\begin{tabular}{ccc}
\hline Load Weight & MAPE & Standard Deviation \\
\hline $250 \mathrm{~kg}$ & $13.98 \%$ & $13.56 \%$ \\
$500 \mathrm{~kg}$ & $9.42 \%$ & $6.78 \%$ \\
\hline
\end{tabular}

The possible sources of the error are listed below:

- The static friction is kept outside of this study and the static friction might be different even for the same static posture with different load weights in the bucket.

- The accuracy of the predicted no load torque is directly related to the gravitational parameters. Increasing the number of static postures in the training data set and covering more parts of the excavator's working space would result in better no load torque estimations; therefore, the error in the load weight estimation would be lowered.

- The used reference loads are concrete blocks. Thus, the load is not uniformly distributed in the bucket. The position of these concrete blocks in the bucket might have an affect on the pressure readings and the measured torque value, $\tau_{23_{L}}$ in the equation (13) might change.

\section{Dynamic estimation of the load weight}

The dynamic torque equations given in the equation (3) can also be written in the decoupled form and using the well-known trigonometric identities $\cos (\alpha+\beta)=\cos (\alpha) \cos (\beta)-\sin (\alpha) \sin (\beta)$ and $\sin (\alpha+\beta)=\sin (\alpha) \cos (\beta)+$ 
$\cos (\alpha) \sin (\beta)$, we can write the dynamic torque equations in matrix form as done in the equation (14). Note that the equation is in the form of $\tau=Y(\Theta, \dot{\Theta}, \ddot{\Theta}) \pi$ as mentioned in the equation (1).

$$
\Delta \tau=\left[\begin{array}{c}
\tau_{4} \\
\tau_{34} \\
\tau_{23}
\end{array}\right]_{3 \times 1}=\left[\begin{array}{ccccccccc}
y_{11} & 0 & 0 & y_{14} & y_{15} & 0 & 0 & 0 & 0 \\
0 & y_{22} & 0 & y_{24} & y_{25} & y_{26} & y_{27} & 0 & 0 \\
0 & 0 & y_{33} & y_{34} & y_{35} & y_{36} & y_{37} & y_{38} & y_{39}
\end{array}\right]_{3 \times 9}\left[\begin{array}{c}
\pi_{d 1} \\
\pi_{d 2} \\
\pi_{d 3} \\
\pi_{s 1} \\
\pi_{s 2} \\
\pi_{s 3} \\
\pi_{s 6} \\
\pi_{s 5} \\
\pi_{s 6}
\end{array}\right]_{9 \times 1}
$$

Where the subscripts s and d stand for static and dynamic parameters, respectively, and

$$
\begin{aligned}
& y_{11}=\ddot{\theta}_{234} \\
& y_{14}=a_{2} \ddot{\theta}_{2} \cos \left(\theta_{34}\right)+a_{2} \dot{\theta}_{2}^{2} \sin \left(\theta_{34}\right)+a_{3} \ddot{\theta}_{23} \cos \left(\theta_{4}\right)+a_{3} \dot{\theta}_{23}^{2} \sin \left(\theta_{4}\right)+g \cos \left(\theta_{234}\right) \\
& y_{15}=-a_{2} \ddot{\theta}_{2} \sin \left(\theta_{34}\right)+a_{2} \dot{\theta}_{2}^{2} \cos \left(\theta_{34}\right)-a_{3} \ddot{\theta}_{23} \sin \left(\theta_{4}\right)+a_{3} \dot{\theta}_{23}^{2} \cos \left(\theta_{4}\right)-g \sin \left(\theta_{234}\right) \\
& y_{22}=\ddot{\theta}_{23} \\
& y_{24}=a_{3} \ddot{\theta}_{234} \cos \left(\theta_{4}\right)-a_{3} \dot{\theta}_{234}^{2} \sin \left(\theta_{4}\right) \\
& y_{25}=-a_{3} \dot{\theta}_{234}^{2} \sin \left(\theta_{4}\right)-a_{3} \dot{\theta}_{234}^{2} \cos \left(\theta_{4}\right) \\
& y_{26}=a_{2} \ddot{\theta}_{2} \cos \left(\theta_{3}\right)+a_{2} \dot{\theta}_{2}^{2} \sin \left(\theta_{3}\right)+g \cos \left(\theta_{23}\right) \\
& y_{27}=-a_{2} \ddot{\theta}_{2} \sin \left(\theta_{3}\right)+a_{2} \dot{\theta}_{2}^{2} \cos \left(\theta_{3}\right)-g \sin \left(\theta_{23}\right) \\
& y_{33}=\ddot{\theta}_{2} \\
& y_{34}=a_{2} \ddot{\theta}_{234} \cos \left(\theta_{34}\right)-a_{2} \dot{\theta}_{234}^{2} \sin \left(\theta_{34}\right) \\
& y_{35}=-a_{2} \ddot{\theta}_{234} \sin \left(\theta_{34}\right)-a_{2} \dot{\theta}_{234}^{2} \cos \left(\theta_{34}\right) \\
& y_{36}=a_{2} \ddot{\theta}_{23} \cos \left(\theta_{3}\right)-a_{2} \dot{\theta}_{23}^{2} \sin \left(\theta_{3}\right) \\
& y_{37}=-a_{2} \ddot{\theta}_{23} \sin \left(\theta_{3}\right)-a_{2} \dot{\theta}_{23}^{2} \cos \left(\theta_{3}\right) \\
& y_{38}=g \cos \left(\theta_{2}\right) \\
& y_{39}=-g \sin \left(\theta_{2}\right)
\end{aligned}
$$

It can be realized from the parameter vector $(\pi)$ that one dynamic parameter $\left(\pi_{d_{i}}\right)$ is introduced for each torque equation and the parameter vector is given in the equation (16) below.

$$
\pi=\left[\begin{array}{c}
\pi_{d 1} \\
\pi_{d 2} \\
\pi_{d 3} \\
\pi_{s 1} \\
\pi_{s 2} \\
\pi_{s 3} \\
\pi_{s 6} \\
\pi_{s 5} \\
\pi_{s 6}
\end{array}\right]=\left[\begin{array}{c}
I_{b u}+M_{b u} r_{4}^{2} \\
I+M_{s t} r_{3}^{2}+M_{b u} a_{3}^{2} \\
I_{b o}+M_{b o} r_{2}^{2}+\left(M_{s t}+M_{b u}\right) a_{2}^{2} \\
M_{b u} r_{4} \cos \left(\alpha_{4}\right) \\
M_{b u} r_{4} \sin \left(\alpha_{4}\right) \\
M_{b u} a_{3}+M_{s t} r_{3} \cos \left(\alpha_{3}\right) \\
M_{s t} r_{3} \sin \left(\alpha_{3}\right) \\
\left(M_{b u}+M_{s t}\right) a_{2}+M_{b o} r_{2} \cos \left(\alpha_{2}\right) \\
M_{b o} r_{2} \sin \left(\alpha_{2}\right)
\end{array}\right]
$$

The torque values have been obtained using the pressure readings and angular position measurements of the machine links as described in the section 4. After constructing the regressor matrix $Y(\Theta, \dot{\Theta}, \ddot{\Theta})$ it is possible to estimate the dynamic parameters using Least Squares Estimation as explained in the section 5.1.

\subsection{Dynamic parameter estimation}

In order to perform the dynamic parameter estimation using the Least Squares Estimation method, five different data sets with empty bucket over the similar trajectories have been collected. In order to avoid the contact force between the bucket and the ground, the trajectories have been generated on free space. 
One of the five data sets have been used for estimating the parameters given in the equation (16), and the other four data sets have been used to compare the predicted torque values that are found using the estimated dynamic parameters with the actual torque values.

In order to construct the regressor matrix $Y(\Theta, \dot{\Theta}, \ddot{\Theta})$, the angular velocity and the angular acceleration values of the boom link, the stick link, and the bucket link are needed as can be seen in the equation (15). Due to the practical limitations, the angular acceleration values could not be recorded; therefore, these values were needed to be estimated. Using a polynomial fitting approach, the angular acceleration values have been estimated. The smoothing splines method have been utilized in MATLAB in order to fit the piece-wise polynomials on the angular position data and the angular velocities and angular accelerations have been found by taking the derivatives of the fitted polynomials. Once the angular velocity estimations matched the angular velocity measurements, the angular acceleration estimations have been assumed to be correct.

The root-mean-squared error (RMSE) values for each link are reported in the table 4 below.

Table 4: Root-mean-squared error values for estimated angular velocities

\begin{tabular}{ccc}
\hline Estimated Angular Velocity & & RMSE (deg/s) \\
\hline Boom velocity & $\dot{\theta}_{2}$ & 0.33 \\
Stick velocity & $\dot{\theta}_{23}$ & 0.35 \\
Bucket velocity & $\dot{\theta}_{234}$ & 0.34 \\
\hline
\end{tabular}

After estimating the angular velocity and angular acceleration values for all the links with acceptable error rates, the regressor matrix $Y(\Theta, \dot{\Theta}, \ddot{\Theta})$ has been constructed and the parameter estimation has been performed on the torque difference between the boom joint and the stick joint, $\tau_{23}$ that is given in the equation (17) below.

$$
\tau_{23}=\left[\begin{array}{c}
\ddot{\theta}_{2} \\
a_{2} \ddot{\theta}_{234} \cos \left(\theta_{34}\right)-a_{2} \dot{\theta}_{234}^{2} \sin \left(\theta_{34}\right) \\
-a_{2} \ddot{\theta}_{234} \sin \left(\theta_{34}\right)-a_{2} \dot{\theta}_{234}^{2} \cos \left(\theta_{34}\right) \\
a_{2} \ddot{\theta}_{23} \cos \left(\theta_{3}\right)-a_{2} \dot{\theta}_{23}^{2} \sin \left(\theta_{3}\right) \\
-a_{2} \ddot{\theta}_{23} \sin \left(\theta_{3}\right)-a_{2} \dot{\theta}_{23}^{2} \cos \left(\theta_{3}\right) \\
g \cos \left(\theta_{2}\right) \\
-g \sin \left(\theta_{2}\right)
\end{array}\right]^{T}\left[\begin{array}{c}
I_{b o}+M_{b o} r_{2}^{2}+\left(M_{s t}+M_{b u}\right) a_{2}^{2} \\
M_{b u} r_{4} \cos \left(\alpha_{4}\right) \\
M_{b u} r_{4} \sin \left(\alpha_{4}\right) \\
M_{b u} a_{3}+M_{s t} r_{3} \cos \left(\alpha_{3}\right) \\
M_{s t} r_{3} \sin \left(\alpha_{3}\right) \\
\left(M_{b u}+M_{s t}\right) a_{2}+M_{b o} r_{2} \cos \left(\alpha_{2}\right) \\
M_{b o} r_{2} \sin \left(\alpha_{2}\right)
\end{array}\right]
$$

Parameter estimation has been performed over 10 seconds of a time interval on one of the five dynamic trajectories. In other words, 2000 data samples have been used. The values of the parameters given in the equation (17) have been found using the formula given in the equation (18), and listed in the table 5 below.

$$
\pi=\left(Y(\Theta, \dot{\Theta}, \ddot{\Theta})^{T} Y(\Theta, \dot{\Theta}, \ddot{\Theta})\right)^{-1} Y(\Theta, \dot{\Theta}, \ddot{\Theta})^{T} \tau_{23}
$$

Table 5: Estimated values of parameters appearing in the dynamic torque difference between the boom and the stick

\begin{tabular}{ccc}
\hline Parameter & Estimated Value & Unit \\
\hline$\pi_{d 3}$ & 28827.71 & $\mathrm{kgm}^{2}$ \\
$\pi_{s 1}$ & 298.04 & $\mathrm{kgm}$ \\
$\pi_{s 2}$ & 592.86 & $\mathrm{kgm}$ \\
$\pi_{s 3}$ & 8228.37 & $\mathrm{kgm}$ \\
$\pi_{s 4}$ & 2571.05 & $\mathrm{kgm}$ \\
$\pi_{s 5}$ & 8927.32 & $\mathrm{kgm}$ \\
$\pi_{s 6}$ & 1386.54 & $\mathrm{kgm}$ \\
\hline
\end{tabular}

Note that all the parameters given in the table 5 above are positive, as expected. It is possible to predict the torque difference between the boom and the stick, $\tau_{23}$, for any position, velocity and acceleration values of the excavator links. Note also that the estimated gravitational parameters are not the same with the results obtained in the static estimation that is discussed in the section 4.1. Since the static friction is eliminated in the dynamic estimation of the parameters, the results of torque predictions on dynamic trajectories are more consistent. In order to check the 
reliability of these parameters, the torque values of the remaining four data sets have been predicted and compared with the actual torque values that are calculated based on the pressure readings and angular position measurements. The mean-absolute-percentage-error (MAPE) values of torque predictions for each data set are listed in the table 6.

Table 6: Accuracy of predicted dynamic no-load torque values

\begin{tabular}{cc}
\hline Predicted $\tau_{23_{N L}}$ & MAPE \\
\hline Data set \#1 & $4.20 \%$ \\
Data set \#2 & $5.24 \%$ \\
Data set \#3 & $5.67 \%$ \\
Data set \#4 & $3.85 \%$ \\
\hline
\end{tabular}

The predicted torque values have an error around 5\%, meaning that the parameters given in the table 5 estimated accurately. Figure 4 illustrates the predicted torque values together with the actual ones.
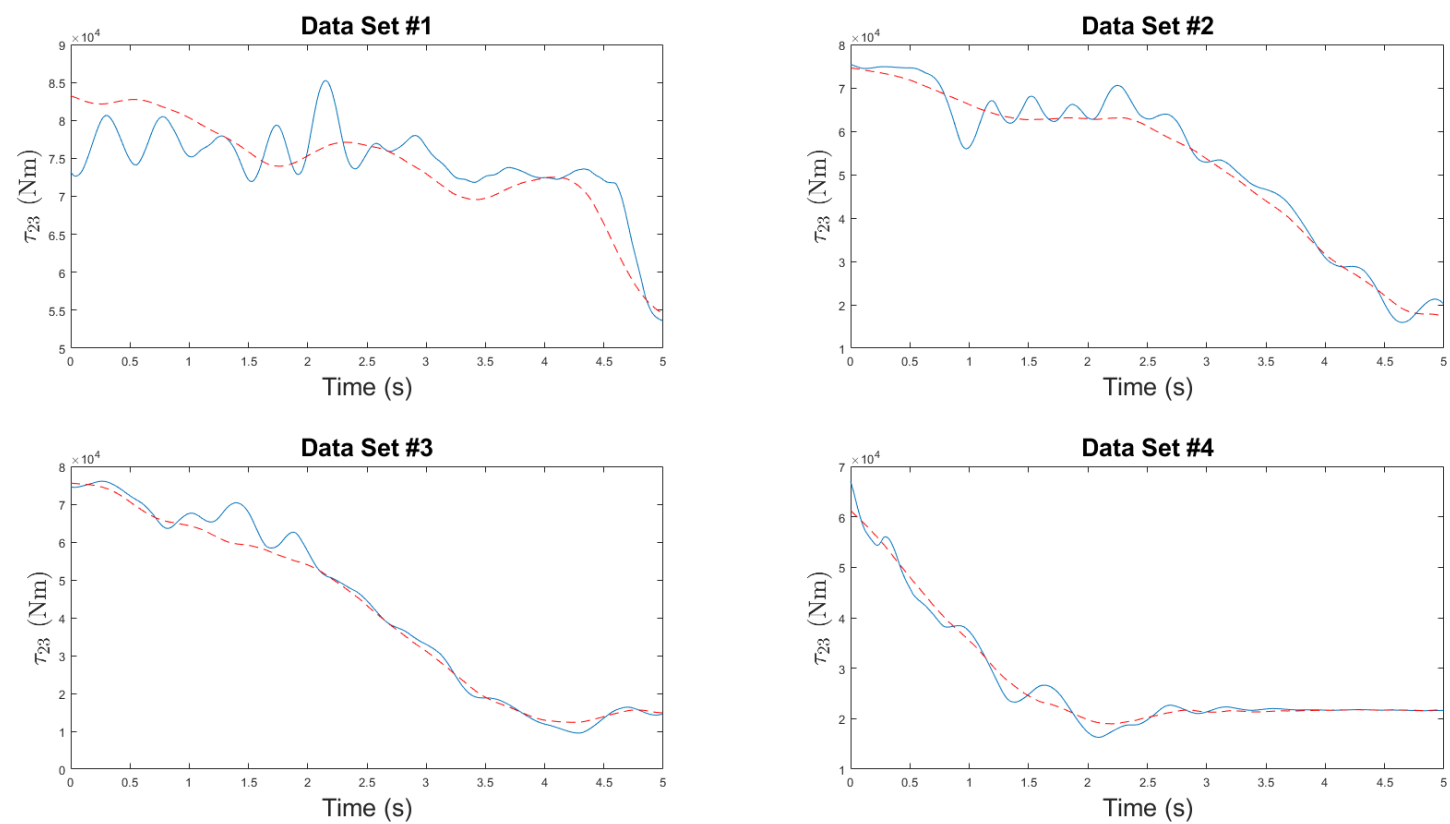

Figure 4: Measured (-) and predicted (-- -) torque values

\subsection{Load weight estimation using the dynamic parameters}

Assuming that the bucket center of gravity is fixed and does not change depending on the variable load weight in the bucket, the dynamic torque equation representing the difference between the boom torque and the stick torque, $\tau_{23}$, can be obtained by replacing $M_{b u}$ with $M_{b u}+\hat{M}$ where $\hat{M}$ is the arbitrary load mass in the bucket as given in the equation 19

$$
\begin{aligned}
\tau_{2_{L}}-\tau_{3_{L}}=\tau_{23_{L}}= & {\left[I_{b o}+M_{b o} r_{2}^{2}+\left(M_{s t}+\left(M_{b u}+\hat{M}\right) a_{2}^{2}\right)\right] \ddot{\theta}_{2} } \\
& +M_{s t} a_{2} r_{3}\left[\ddot{\theta}_{23} \cos \left(\theta_{3}+\alpha_{3}\right)-\dot{\theta}_{23}^{2} \sin \left(\theta_{3}+\alpha_{3}\right)\right] \\
& +\left(M_{b u}+\hat{M}\right) a_{2} a_{3}\left(\ddot{\theta}_{23} \cos \left(\theta_{3}\right)-\dot{\theta}_{23}^{2} \sin \left(\theta_{3}\right)\right. \\
& +\left(M_{b u}+\hat{M}\right) a_{2} r_{4}\left[\ddot{\theta}_{234} \cos \left(\theta_{34}+\alpha_{4}\right)-\dot{\theta}_{234}^{2} \sin \left(\theta_{34}+\alpha_{4}\right)\right] \\
& +\left(M_{b u}+\hat{M}+M_{s t}\right) g a_{2} \cos \left(\theta_{2}\right)+M_{b o} g r_{2} \cos \left(\theta_{2}+\alpha_{2}\right)
\end{aligned}
$$

The equation (19) above can be rearranged as the summation of the no load torque and the torque due to the load weight in the bucket. The resulting torque equation is given in the equation (20) below. 


$$
\begin{aligned}
\tau_{23_{L}}= & \tau_{23_{N L}}+\hat{M} a_{2}^{2} \ddot{\theta}_{2}+\hat{M} a_{2} a_{3}\left(\ddot{\theta}_{23} \cos \left(\theta_{3}\right)-\dot{\theta}_{23}^{2} \sin \left(\theta_{3}\right)\right) \\
& +\hat{M} r_{4} a_{2} \ddot{\theta}_{234} \cos \left(\theta_{34}+\alpha_{4}\right)-\hat{M} r_{4} a_{2} \dot{\theta}_{234}^{2} \sin \left(\theta_{34}+\alpha_{4}\right)+\hat{M} g a_{2} \cos \left(\theta_{2}\right)
\end{aligned}
$$

The torque value with the load $\left(\tau_{23_{L}}\right)$ in the bucket is calculated using the pressure measurements from the cylinders and the angular position measurements of the links, as discussed earlier. Furthermore, the no load torque value $\left(\tau_{23_{N L}}\right)$ can be predicted for the same angular position, velocity and acceleration values. Then, $\hat{M}$ can be found using the difference between $\tau_{23_{L}}$ and $\tau_{23_{N L}}$ as stated in the equation (21) below [1].

$$
\hat{M}=\frac{\tau_{23_{L}}-\tau_{23_{N L}}}{a_{2}^{2} y_{33}+r_{4} \cos \left(\alpha_{4}\right) y_{34}+r_{4} \sin \left(\alpha_{4}\right) y_{35}+a_{3} y_{36}+g a_{2} \cos \left(\theta_{2}\right)}
$$

Where the parameters $y_{33}, y_{34}, y_{35}$, and $y_{36}$ are given in the equation (15).

It should be noted that the polar coordinates of the bucket center of gravity, $r_{4}$ and $\alpha_{4}$ that can be seen in the figure 2 are needed in order to estimate the payload mass in the bucket that is given in the equation (21) above. Estimation of these parameters is discussed in the section 5.2.1.

\subsubsection{Estimation of the polar coordinates of the bucket center of gravity}

In order to estimate the polar coordinates of the bucket center of gravity, one can use the second and the third elements of the dynamic parameter vector that is described in the equation (16), $\pi_{s 1}$ and $\pi_{s 2}$, together with the mass of the bucket and tiltrotator assembly. Knowing the fact that the bucket-tiltrotator is $750 \mathrm{~kg}$, the parameter $\alpha_{4}$ can be found taking the inverse tangent of the division of $\pi_{s 2}$ to $\pi_{s 21}$ gives us the parameter $\alpha_{4}$ as shown in the equation (22) below.

$$
\alpha_{4}=\tan ^{-1}\left(\frac{\pi_{s 2}}{\pi_{s 1}}\right)
$$

Once the value of $\alpha_{4}$ is obtained, the parameter $r_{4}$ can be found using one of the following relations given in the equation (23) below.

$$
\begin{aligned}
r_{4} & =\frac{\pi_{s 1}}{M_{b u} \cos \left(\alpha_{4}\right)} \\
r_{4} & =\frac{\pi_{s 2}}{M_{b u} \sin \left(\alpha_{4}\right)}
\end{aligned}
$$

Once the values of the parameters $\alpha_{4}$ and $r_{4}$ have been obtained, it becomes possible to estimate the load weight in the bucket using the equation (21) above.

\subsection{Collected data sets and the results}

In order to find out the usability of the method developed, five data sets with $318 \mathrm{~kg}$ reference load and five data sets with $618 \mathrm{~kg}$ reference load have been collected using similar trajectories on free space. The mass estimation formula given in the equation (21) has been used for every sample in the used data sets and an array of mass estimation is created and the mean value is reported as the final result of the estimated load weight.

Three scenarios have been generated and the load weight estimation has been performed in each scenario that are listed below:

- Load weight estimation over five seconds of time intervals where the machine links have velocities higher than $2 \mathrm{deg} / \mathrm{s}$ in magnitude.

- Load weight estimation using the dynamic parts of the data sets, i.e. the data samples when the machine links are stationary discarded.

- Load weight estimation using all the samples in the data sets including the stationary parts as well.

The load weight estimation for these three scenarios stated above are presented in the tables 7,8 and 9 , respectively. 
Table 7: The results of dynamic load weight estimation over 5 seconds of time intervals

\begin{tabular}{ccc}
\hline Test Data & Estimated Load Weight & Error (\%) \\
\hline Data set \#1, 618 kg & $614.22 \mathrm{~kg}$ & $0.61 \%$ \\
Data set \#2,618 kg & $612.39 \mathrm{~kg}$ & $0.91 \%$ \\
Data set \#3,618 kg & $610.89 \mathrm{~kg}$ & $1.15 \%$ \\
Data set \#4, 618 kg & $623.53 \mathrm{~kg}$ & $0.90 \%$ \\
Data set \#5, 618 kg & $621.33 \mathrm{~kg}$ & $0.54 \%$ \\
Data set \#1, 318 kg & $319.24 \mathrm{~kg}$ & $0.39 \%$ \\
Data set \#2,318 kg & $313.99 \mathrm{~kg}$ & $1.26 \%$ \\
Data set \#3, 318 kg & $317.19 \mathrm{~kg}$ & $0.26 \%$ \\
Data set \#4, 318 kg & $321.68 \mathrm{~kg}$ & $1.16 \%$ \\
Data set \#5, 318 kg & $332.69 \mathrm{~kg}$ & $4.62 \%$ \\
\hline
\end{tabular}

Table 8: The results of dynamic load weight estimation using only the dynamic parts of the data sets

\begin{tabular}{ccc}
\hline Test Data & Estimated Load Weight & Error (\%) \\
\hline Data set \#1, 618 kg & $630.77 \mathrm{~kg}$ & $2.07 \%$ \\
Data set \#2,618 kg & $642.37 \mathrm{~kg}$ & $3.94 \%$ \\
Data set \#3,618 kg & $621.90 \mathrm{~kg}$ & $0.63 \%$ \\
Data set \#4, 618 kg & $606.41 \mathrm{~kg}$ & $1.87 \%$ \\
Data set \#5, 618 kg & $612.75 \mathrm{~kg}$ & $0.85 \%$ \\
Data set \#1, 318 kg & $336.17 \mathrm{~kg}$ & $5.71 \%$ \\
Data set \#2, 318 kg & $310.63 \mathrm{~kg}$ & $2.32 \%$ \\
Data set \#3, 318 kg & $310.84 \mathrm{~kg}$ & $2.25 \%$ \\
Data set \#4, 318 kg & $349.36 \mathrm{~kg}$ & $9.86 \%$ \\
Data set \#5, 318 kg & $323.26 \mathrm{~kg}$ & $1.65 \%$ \\
\hline
\end{tabular}

Table 9: The results of dynamic load weight estimation using all the samples in the data sets

\begin{tabular}{ccc}
\hline Test Data & Estimated Load Weight & Error (\%) \\
\hline Data set \#1, 618 kg & $618.87 \mathrm{~kg}$ & $0.14 \%$ \\
Data set \#2,618 kg & $510.55 \mathrm{~kg}$ & $17.39 \%$ \\
Data set \#3, 618 kg & $603.33 \mathrm{~kg}$ & $2.37 \%$ \\
Data set \#4, 618 kg & $567.38 \mathrm{~kg}$ & $8.19 \%$ \\
Data set \#5, 618 kg & $541.41 \mathrm{~kg}$ & $12.39 \%$ \\
Data set \#1, 318 kg & $279.24 \mathrm{~kg}$ & $12.19 \%$ \\
Data set \#2, 318 kg & $298.28 \mathrm{~kg}$ & $6.20 \%$ \\
Data set \#3, 318 kg & $290.09 \mathrm{~kg}$ & $8.78 \%$ \\
Data set \#4, 318 kg & $340.66 \mathrm{~kg}$ & $7.13 \%$ \\
Data set \#5, 318 kg & $301.64 \mathrm{~kg}$ & $5.15 \%$ \\
\hline
\end{tabular}


As can be seen from the results, the best results are achieved with high velocity values and the worst results are obtained with the inclusion of the stationary parts of the data sets. The possible sources of the errors are listed below:

- The frictional parameters are neglected in dynamic modeling but the effects of the friction was present in the data sets.

- Used reference load weights were concrete blocks; therefore, the center of gravity has changed with different load weights in the bucket but it assumed to be unchanged.

- The accuracy of the dynamic parameters have an effect on the torque predictions and more accurate estimations would yield more accurate torque predictions.

- Angular velocity estimations and angular acceleration estimations were not $100 \%$ accurate. Therefore, the predicted torque values have been affected and resulted in an increase for the error of the dynamic load weight estimations.

\section{Conclusion}

We presented solutions for load weight estimation problem on excavators. We proposed two different methods based on static and dynamic models. The dynamic model of the excavator is developed considering the excavator as a planar robot manipulator with three-revolute joints: the boom, the stick and the bucket. The cabin frame is kept outside the scope of this research and the tiltrotator is assumed to be non-functioning and only a series of offsets. Due to the practical limitations, we were not able to record the angular acceleration measurements and we estimated these values using a polynomial fitting approach. Even though the friction is neglected throughout the study for simplicity, effects of the static friction was present in the data and it was observed in the results of static estimation of the load weight. A dynamic load weight estimation method is proposed assuming that the bucket center of gravity is independent from the load mass in the bucket and the results show that the approach can work accurately with high velocity motion trajectories. The proposed method for dynamic estimation of the load weight can be implemented real-time if the angular acceleration values can be measured together with the angular position and angular velocity values. Accurate results could be obtained real-time when the friction is not dominating. Also, the idea of dynamic parameter estimation and using the torque difference between the case when the end effector carries load and the case when the end effector is unloaded could be extended to other machines such as cranes but needs further investigation. Finally, the possible sources of the error for the estimated load weights have been discussed.

\section{References}

[1] Mehmet Ferlibas. Load weight estimation on excavators. Master's thesis, Tampere University, 102020.

[2] Jahmy J. Hindman. Dynamic payload estimation in four wheel drive loaders. PhD thesis, University of Saskatchewan, 2008.

[3] Frederic Ballaire. Dynamic, continuous, and center of gravity independent weighing with a loader. $\mathrm{PhD}$ thesis, Technical University of Kaiserslautern, 2015.

[4] S. Tafazoli, P. D. Lawrence, S. E. Salcudean, D. Chan, S. Bachmann, and C. W. de Silva. Parameter estimation and actuator friction analysis for a mini excavator. In IEEE Int. Conf. Robot. Automat., pages 329-334, Minneapolis,MN,USA, 41996.

[5] Artur Gawlik and Piotr Kucybała. Dynamic weighing system used in excavator. Journal of KONES, 24(4):31 $-38,2017$.

[6] Anton Renner, Hannes Wind, and Oliver Sawodny. Online payload estimation for hydraulically actuated manipulators. Mechatronics, 66:102322, 2020.

[7] Ashwin Walawalkar, S. Heep, Martin Frank, R. Leifeld, and Christian Schindler. Validation of an analytical method for payload estimation in excavators. In Commercial Vehicle Technology 2018, 032018.

[8] Ashwin Walawalkar, Steffen Heep, Florian Schneider, Jan Schüßler, and Christian Schindler. A method for payload estimation in excavators. Proceedings of the 4th Commercial Vehicle Symposium, pages 424-437, 2016. 
[9] Nureddin Bennett, Ashwin Walawalkar, and Christian Schindler. Payload estimation in excavators: Modelbased evaluation of current payload estimation systems. 032014.

[10] N. G. Hareesha and K. N. Umesh. Kinematic and isotropic properties of excavator mechanism. 072018.

[11] Seungjin Yoo, Cheol-Gyu Park, and Seung-Han You. Inertial parameter estimation for the dynamic simulation of a hydraulic excavator. Journal of Mechanical Science and Technology, 32(9):4045-4056, 2018.

[12] Yahya H. Zweiri. Identification schemes for unmanned excavator arm parameters. 5(2):185-192, 2008.

[13] Shahram Tafazoli, Peter. D. Lawrence, and S.E. Salcudean. Identification of inertial and friction parameters for excavator arms. IEEE Transactions on Robotics and Automation, 15(5):966-971, 101999.

[14] Shahram Tafazoli. Identification of frictional effects and structural dynamics for improved control of hydraulic excavators. PhD thesis, University of British Columbia, 1997.

[15] Yunfei Dong, Tianyu Ren, Ken Chen, and Dan Wu. An efficient robot payload identification method for industrial application. Industrial robot, 45(4):505-515, 2018.

[16] N. R. Parker, S. E. Salcudean, and P. D. Lawrence. Application of force feedback to heavy duty hydraulic machines. In IEEE Int. Conf. Robot. Automat., pages 375-381, 51993.

[17] Bruno Siciliano, Lorenzo Sciavicco, Luigi Villani, and Giuseppe Oriolo. Robotics Modelling, Planning and Control, chapter 7. Springer, 2009. 